\title{
HIGHER ORDER $Z$-IDEALS IN COMMUTATIVE RINGS
}

\author{
THEMBA DUBE AND OGHENETEGA IGHEDO
}

Received 23 May, 2015

\begin{abstract}
We study ideals that resemble $z$-ideals in commutative rings with identity. For each positive integer $n$, we say an ideal of a commutative ring $A$ is a $z^{n}$-ideal in case it has the property that if $a$ and $b$ belong to the same maximal ideals of $A$, and $a^{n} \in I$, then $b^{n}$ is also in $I$. The set of all $z^{n}$-ideals of $A$ is denoted by $\mathfrak{Z}^{n}(A)$. This gives an ascending chain $\mathfrak{Z}(A) \subseteq \mathfrak{Z}^{2}(A) \subseteq$ $\mathfrak{Z}^{3}(A) \subseteq \cdots$ of collections of ideals, starting with the collection of $z$-ideals. We give examples of when the chain becomes stationary, and when it ascends without stop, with each collection properly contained in its successor. The assignment $A \mapsto \mathfrak{Z}^{n}(A)$ is shown to be the object part of a functor $\mathrm{Rng}_{\mathfrak{z}}^{\text {op }} \rightarrow$ Set, where Rng $\mathrm{z}_{\mathfrak{z}}$ denotes the category of commutative rings with ring homomorphisms that contract $z$-ideals to $z$-ideals. When the objects are restricted to rings with zero Jacobson radical, the restricted functor reflects epimorphisms, but not monomorphisms.
\end{abstract}

2010 Mathematics Subject Classification: 13A15; 13B30

Keywords: commutative ring, $z$-ideal, higher order $z$-ideal, subfunctor, monomorphism, epimorphism

\section{INTRODUCTION}

Throughout the paper all rings are commutative with identity. The notion of $z$ ideal was first studied by Kohls [12] in the rings $C(X)$ of real-valued continuous functions on a Tychonoff space $X$. Although he defined these ideals topologically, in terms of zero-sets, he showed that they can be characterized algebraically. As is well documented in the classical text of Gillman and Jerison [7], $z$-ideals have proved to be a powerful tool in the study of both algebraic properties of function rings, and topological properties of Tychonoff spaces.

In 1980 and 1981, $z$-ideals were introduced in the context of Riesz spaces by Huijsman and de Pagter (see [8], [9] and [5]). They showed that in the Riesz space $C(X)$ the $z$-ideals, as they defined them, were precisely those of Kohls. It was Mason [15] who initiated the study of $z$-ideals in arbitrary commutative rings with identity. In [14], Larson studies instances where the sum of $z$-ideals (and other types of ideals) are $z$-ideals, as is the case in rings of continuous functions.

The authors were supported by the National Research Foundation of South Africa via a grant with Grant No. 93514. 
In this paper we tweak slightly Mason's definition of $z$-ideal by considering powers of elements. We thus obtain, for each positive integer $n$, a generalization of $z$-ideals. The resulting ideals we call $z^{n}$-ideals. For any ring $A$, we denote the set of $z^{n}$ ideals of $A$ by $\mathfrak{Z}^{n}(A)$. It turns out that, for every positive integer $n, \mathfrak{Z}^{n}(A) \subseteq \mathfrak{Z}^{n+1}$ (Lemma 1), which then leads to the ascending chain

$$
\mathfrak{Z}(A) \subseteq \mathfrak{Z}^{2}(A) \subseteq \mathfrak{Z}^{3}(A) \subseteq \cdots,
$$

which we call the $z$-tower of $A$. There are instances where the $z$-tower becomes stationary (Examples 1, 2, 3, and 4), and instances where it does not (Example 5). If the $z$-tower of $A$ becomes stationary, we say $A$ is a $z$-terminating ring. Lying above all the collections $\mathfrak{Z}^{n}(A)$ is the collection $\mathfrak{Z}^{\text {rad }}(A)$ of what are called $\sqrt{z}$-ideals in [2]. These are ideals $I$ for which the radical of $I$ is a $z$-ideal. We say $A$ is radically $z$ covered if $\bigcup_{n=1}^{\infty} \mathfrak{Z}^{n}(A)=\mathfrak{Z}^{\mathrm{rad}}(A)$. Although there are Noetherian rings which are not $z$-terminating (for instance the ring of integers, as shown in Example 5), we prove in Theorem 1 that every Noetherian ring is radically $z$-covered.

Turning to products, we show that the direct product of finitely many rings is $z$ terminating if and only if each factor is $z$-terminating (Theorem 2), and the direct product of finitely many rings is radically $z$-covered if and only if each factor is radically $z$-covered (Proposition 2).

In the last section we show that, for each positive integer $n$, the assignment $A \mapsto$ $\mathfrak{Z}^{n}(A)$ is the object part of a functor $\mathrm{Rng}_{\mathfrak{z}}^{\text {op }} \rightarrow$ Set, where Rng $\mathrm{z}_{\mathfrak{z}}$ denotes the category of commutative rings with ring homomorphisms that contract $z$-ideals to $z$-ideals. The functor sends a morphism $\tau$ in $\operatorname{Rng}_{\mathfrak{z}}^{\text {op }}$ to $\varrho^{-1}$, where $\varrho$ is the ring homomorphism determining $\tau$. We show (Theorem 3 ) that the restriction of this functor to the subcategory of Rng ${ }_{\mathfrak{z}}{ }^{\text {op }}$ whose objects are the rings with zero Jacobson radical reflects epimorphisms. We give an example to show that it does not reflect monomorphisms.

\section{PRELIMINARIES AND NOTATION}

Let us reiterate that by the term "ring" we mean a commutative ring with identity. We do not insist that our ideals be proper, so the whole ring will be considered as an ideal. On the other hand though, as is tradition, every prime ideal (and hence every maximal ideal) is a proper ideal. One of the consequences of insisting that our rings have identity is that if $A$ and $B$ are rings, then every ideal of the direct product $A \times B$ is of the form $I \times J$, for some ideals $I$ and $J$ of $A$ and $B$ respectively. It is of course possible for rings $A$ and $B$ which do not have identity to have the property that every ideal of $A \times B$ is of the form $I \times J$, for some ideals $I$ and $J$ of $A$ and $B$ respectively (see [1, Proposition 1]).

For any $a \in A$, we denote by $\mathfrak{M}(a)$ the set of all maximal ideals of $A$ containing $a$. An ideal $I$ of a ring $A$ is a $z$-ideal if, for any $a, b \in A, \mathfrak{M}(a) \supseteq \mathfrak{M}(b)$ and $b \in I$ imply $a \in I$. The containment in this definition can be replaced with an equality. The 
Jacobson radical of $A$ will be denoted by $\operatorname{Jac}(A)$. A ring is reduced if 0 is its only nilpotent element. If $\phi: A \rightarrow B$ is a ring homomorphism, and $I$ is an ideal of $B$, then the ideal $\phi^{-1}[I]$ is called the contraction of $I$.

We shall have occasion to refer to $f$-rings. Our reference for these rings is [18]. Recall that an ideal $I$ of an $f$-ring is an $\ell$-ideal if whenever $|a| \leq|b|$ and $b \in I$, then $a \in I$. An $f$-ring $A$ is 1 -convex if for any $u, v \in A$ such that $0 \leq u \leq v$, there is a $w \in A$ such that $u=v w$. In [13, Theorem 2.4], Larson proves that in a reduced 1convex $f$-ring every ideal is an $\ell$-ideal. An $f$-ring $A$ has bounded inversion if every $a \in A$ with $a \geq 1$ is invertible. For reduced $f$-rings, this holds precisely when every maximal ideal in $A$ is an $\ell$-ideal.

\section{A TOWER OF $z$-LIKE IDEALS}

We start by defining the ideals that will form the main study in this paper.

Definition 1. Let $n$ be a positive integer. An ideal $I$ of a ring $A$ is a $z^{n}$-ideal if, for any $a, b \in A$,

$$
\mathfrak{M}(a) \supseteq \mathfrak{M}(b) \text { and } b^{n} \in I \quad \Longrightarrow \quad a^{n} \in I .
$$

We denote by $\mathfrak{Z}^{n}(A)$ the set of all $z^{n}$-ideals of $A$. In particular, $\mathfrak{Z}(A)$ denotes the set of all $z$-ideals of $A$.

It is clear that every $z$-ideal is a $z^{n}$-ideal for every $n \in \mathbb{N}$, so that, indeed, these ideals generalize $z$-ideals in a natural way. They can also be characterized similarly to $z$-ideals in terms of intersections of maximal ideals. Let us expatiate. Let $A$ be a ring, $a$ be an element of $A$, and $n$ be a positive integer. We set

$$
M(a)=\bigcap \mathfrak{M}(a) \quad \text { and } \quad M^{n}(a)=\left\{x^{n} \mid x \in M(a)\right\} ;
$$

with the convention that if $a$ is contained in no maximal ideal, then $M^{n}(a)=A$. It is clear that $M^{n}(a) \subseteq M(a)$ for every $n$. The proofs of the following characterizations are fairly routine.

Proposition 1. Let $A$ be a ring, and $n$ be a positive integer. The following are equivalent for an ideal I of $A$.

(1) I is a $z^{n}$-ideal.

(2) For any $a, b \in A$, if $\mathfrak{M}(a)=\mathfrak{M}(b)$ and $b^{n} \in I$, then $a^{n} \in I$.

(3) For any $a \in I, M^{n}(a) \subseteq I$.

Remark 1. In [15, Theorem 1.2], Mason proves that a $\operatorname{ring} A$ is von Neumann regular if and only if every ideal of $A$ is a $z$-ideal. Generalizing von Neumann regular rings, McCoy [16] defines a ring $A$ (not necessarily commutative) to be $\pi$-regular if, for every $a \in A$, there exists an element $x \in A$ and a positive integer $n$ (depending on $a$ ) such that $a^{n} x a^{n}=a^{n}$. If, however, there is a fixed positive integer $m$ such that for every $a \in A$ there exists $x \in A$ for which $a^{m}=a^{m} x a^{m}$, then he says $A$ is 
$m$-regular. Now, going back to the commutative case, if $m$ is a positive integer and every principal ideal of $A$ is a $z^{m}$-ideal, then $A$ is $m$-regular. For, given any $a \in A$, the principal ideal $\left\langle a^{2 m}\right\rangle$ is a $z^{m}$-ideal containing $\left(a^{2}\right)^{m}$. Since $\mathfrak{M}(a)=\mathfrak{M}\left(a^{2}\right)$, it follows that $a^{m} \in\left\langle a^{2 m}\right\rangle$, which implies $a^{m}=a^{2 m} x$, for some $x \in A$, showing that $A$ is $m$-regular.

The following lemma records some elementary observations regarding $z^{n}$-ideals. Recall that the radical of an ideal $I$ of a ring $A$ is the ideal

$$
\sqrt{I}=\left\{a \in A \mid a^{n} \in I \text { for some } n \in \mathbb{N}\right\} .
$$

An ideal $I$ is called a radical ideal if $I=\sqrt{I}$. The set of radical ideals of $A$ will be denoted by $\operatorname{Rad}(A)$. Following [2], we say $I$ is a $\sqrt{z}$-ideal in case $\sqrt{I}$ is a $z$-ideal. We define the set

$$
\mathfrak{Z}^{\mathrm{rad}}(A)=\{I \subseteq A \mid I \text { is a } \sqrt{z} \text {-ideal }\} .
$$

It is easy to see that every $z$-ideal is a radical ideal, so that $\operatorname{Rad}(A) \cap \mathfrak{Z}(A)=\mathfrak{Z}(A)$.

Lemma 1. Let $A$ be a ring, and $n$ be a positive integer. Then we have the following.

(1) $\mathfrak{Z}^{n}(A) \subseteq \mathfrak{Z}^{n+1}(A)$.

(2) $\mathfrak{Z}^{n}(A) \subseteq \mathfrak{Z}^{\operatorname{rad}}(A)$.

(3) $\operatorname{Rad}(A) \cap \mathfrak{Z}^{n}(A)=\mathfrak{Z}(A)$.

Proof. (1) Let $I \in \mathfrak{Z}^{n}(A)$. Consider any $a, b \in A$ with $\mathfrak{M}(a)=\mathfrak{M}(b)$ and $b^{n+1} \in$ $I$. Since $2 n \geq n+1$, we have $\left(b^{2}\right)^{n}=b^{2 n} \in I$. Putting $c=b^{2}$, we see that $a$ and $c$ are elements of $A$ such that $\mathfrak{M}(a)=\mathfrak{M}(c)$ and $c^{n} \in I$. Since $I \in \mathfrak{Z}^{n}(A)$, it follows that $a^{n} \in I$, hence $a^{n+1} \in I$. Therefore $I \in \mathfrak{Z}^{n+1}(A)$, showing that $\mathfrak{Z}^{n}(A) \subseteq \mathfrak{Z}^{n+1}(A)$.

(2) Let $I \in \mathfrak{Z}^{n}(A)$. Consider any $a, b \in A$ such that $\mathfrak{M}(a)=\mathfrak{M}(b)$ and $b \in \sqrt{I}$. Pick $m \in \mathbb{N}$ such that $b^{m} \in I$. Then $\left(b^{m}\right)^{n} \in I$. Since $\mathfrak{M}(a)=\mathfrak{M}\left(b^{m}\right)$ and $I \in$ $\mathfrak{Z}^{n}(A)$, it follows that $a^{n} \in I$. But this implies $a \in \sqrt{I}$; so $\sqrt{I}$ is a $z$-ideal. Thus, $I \in \mathfrak{Z}^{\mathrm{rad}}(A)$, which establishes the desired inclusion.

(3) Since every $z$-ideal is radical, it suffices to show that $\operatorname{Rad}(A) \cap \mathfrak{Z}^{n}(A) \subseteq \mathfrak{Z}(A)$. So let $I$ be a radical $z^{n}$-ideal. Consider any $a, b \in A$ such that $\mathfrak{M}(a)=\mathfrak{M}(b)$ and $b \in I$. Then $b^{n} \in I$, which implies $a^{n} \in I$ since $I$ is a $z^{n}$-ideal, by hypothesis. But $I$ is also a radical ideal, so $a \in I$, which shows that $I$ is a $z$-ideal.

Emanating from part (1) of the preceding lemma, we have the ascending chain

$$
\mathfrak{Z}(A) \subseteq \mathfrak{Z}^{2}(A) \subseteq \mathfrak{Z}^{3}(A) \subseteq \cdots \subseteq \mathfrak{Z}^{n}(A) \subseteq \mathfrak{Z}^{n+1}(A) \subseteq \cdots
$$

of collections of ideals of $A$. We call it the z-tower of $A$. For brevity, we write

$$
\mathfrak{Z}^{\infty}(A)=\bigcup_{n=1}^{\infty} \mathfrak{Z}^{n}(A),
$$


and observe that $\mathfrak{Z}^{\infty}(A) \subseteq \mathfrak{Z}^{\text {rad }}(A)$. We say an ideal of $A$ is a higher order $z$-ideal if it belongs to $\mathfrak{Z}^{\infty}(A)$. If there is a positive integer $k$ such that

$$
\mathfrak{Z}^{k}(A)=\mathfrak{Z}^{k+1}(A)=\mathfrak{Z}^{k+2}(A)=\cdots,
$$

we say the $z$-tower terminates.

Modifying the proof of [7, Theorem 14.7], Mason [15] proves that any prime ideal that is minimal over a $z$-ideal is itself a $z$-ideal. Now, observe that if $P$ is a prime ideal that is minimal over $I$, then $P$ is also minimal over $\sqrt{I}$. Combining this with Mason's result, we deduce from Lemma 1 the following corollary.

Corollary 1. A prime ideal that is minimal over a higher order z-ideal is a z-ideal.

We give a name to rings with terminating $z$-towers.

Definition 2. A ring $A$ is $z$-terminating in case its $z$-tower terminates.

Here are examples of $z$-terminating rings, and of non-z-terminating rings.

Example 1. Every von Neumann regular ring is $z$-terminating. This is so because, as remarked above, a ring is von Neumann regular if and only if every ideal in it is a $z$-ideal.

Example 2. In [17, Corollary 2.5], Mulero proves that an ideal of any $C(X)$ is a $z$-ideal if and only if its radical is a $z$-ideal. Consequently, if $I \in \mathfrak{Z}^{\mathrm{rad}}(C(X))$, so that $\sqrt{I}$ is a $z$-ideal, then $I \in \mathfrak{Z}(C(X))$. Thus, in view of Lemma 1 ,

$$
\mathfrak{Z}(C(X))=\mathfrak{Z}^{2}(C(X))=\mathfrak{Z}^{3}(C(X))=\cdots=\mathfrak{Z}^{\mathrm{rad}}(C(X)),
$$

which shows that every $C(X)$ is a $z$-terminating ring; and, in fact, that the $z$-tower terminates at its base.

Example 3. This example gives a class of $z$-terminating rings which contains properly the class of the rings $C(X)$. We will not recall the required background, and refer the interested reader to [3]. In [6], a ring $A$ for which $\mathfrak{Z}^{\mathrm{rad}}(A)=\mathfrak{Z}(A)$ is called $z$ good. It is then shown in Lemma 3.4 of that paper that $\mathcal{R} L$, the ring of real-valued continuous functions on a completely regular frame $L$, is $z$-good. Therefore each $\mathcal{R} L$ is $z$-terminating. This then covers the $C(X)$ case since every $C(X)$ is isomorphic to some $\mathcal{R} L$. It also transcends the $C(X)$ case because there are frames $L$ for which $\mathcal{R} L$ is not isomorphic to any $C(X)$ (see [3]). It is apposite to remark here that the rings $\mathcal{R} L$ are, up to isomorphism, precisely the homomorphic images of the rings $C(X)$ under $\ell$-ring homomorphisms. See [4] for details.

In the example that follows we say a reduced $f$-ring has square roots if for every $u \geq 0$ there exists a (necessarily unique) $v \geq 0$ such that $v^{2}=u$. In this case we write $u^{\frac{1}{2}}$. For any positive integer $k, u^{\frac{1}{2^{k}}}$ has the obvious meaning. Examples of such $f$-rings are uniformly complete $f$-algebras (see [10, Theorem 3.9]). 
Example 4. If a reduced 1-convex $f$-ring with bounded inversion has square roots, then it is $z$-terminating. We show that in such an $f$-ring every higher order $z$-ideal is a $z$-ideal. Let $n$ be a positive integer, and let $I$ be a $z^{n}$-ideal in an $f$-ring $A$ of the stated kind. Consider any $a, b \in A$ such that $\mathfrak{M}(a)=\mathfrak{M}(b)$ and $b \in I$. Choose $k \in \mathbb{N}$ such that $2^{k} \geq n$. Since $A$ has square roots, $|a|^{\frac{1}{2^{k}}}$ exists in $A$, and, furthermore, $\mathfrak{M}\left(|a|^{\frac{1}{2^{k}}}\right)=\mathfrak{M}(b)$. Since $b^{2^{k}} \in I$, and $I$ is a $z^{2^{k}}$-ideal, by Lemma 1 , it follows that $|a|=\left(|a|^{\frac{1}{2^{k}}}\right)^{2^{k}} \in I$. Since $I$ is an $\ell$-ideal, this implies $a \in I$, showing that $I$ is a $z$-ideal. Consequently, $\mathfrak{Z}^{n}(A)=\mathfrak{Z}(A)$ for every $n$, and hence $A$ is $z$-terminating.

Example 5. The ring of integers is not $z$-terminating. To see this, recall that maximal ideals of $\mathbb{Z}$ are exactly the principal ideals $\langle p\rangle$, for $p$ a prime number. Thus, if $k, l \in \mathbb{N}$ and $\mathfrak{M}(k)=\mathfrak{M}(l)$, then $k$ and $l$ are divisible by exactly the same prime numbers. Hence, if $k$ is even then so is $l$. We claim that for any positive integer $n$, $\mathfrak{Z}^{n}(\mathbb{Z}) \subset \mathfrak{Z}^{n+1}(\mathbb{Z})$, where $\subset$ designates proper containment. Let $I=\left\langle 2^{n+1}\right\rangle$. We will show that $I \in \mathfrak{Z}^{n+1}(\mathbb{Z})$, but $I \notin \mathfrak{Z}^{n}(\mathbb{Z})$. For the former, suppose that $k$ and $l$ are integers with $\mathfrak{M}(k)=\mathfrak{M}(l)$ and $k^{n+1} \in I$. Without loss of generality, we may assume that $k$ and $l$ are positive. Then $k$ is a multiple of $2^{n+1}$, which makes it even, and hence $l$ is even as well, and consequently $l^{n+1}$ is divisible by $2^{n+1}$, that is, $l \in I$. This proves that $I \in \mathfrak{Z}^{n+1}(\mathbb{Z})$. Next, observe that $4^{n} \in I$ because $4^{n}=2^{n-1} \cdot 2^{n+1}$. Also, $\mathfrak{M}(2)=\mathfrak{M}(4)$, but $2^{n} \notin I$ since $2^{n}$ is not an integral multiple of $2^{n+1}$. This shows that $I \notin \mathfrak{Z}^{n}(\mathbb{Z})$.

Remark 2. Denote by $\mathfrak{L}(A)$ the set of $\ell$-ideals of an $f$-ring $A$. If $A$ is 1 -convex, then of course $\mathfrak{Z}^{\text {rad }}(A) \subseteq \mathfrak{L}(A)$. This containment can be proper. Indeed, let $X$ be an $F$-space which is not a $P$-space (see [7] for definitions). Then $C(X)$ is a 1-convex $f$-ring which is not von Neumann regular. Therefore $\mathfrak{Z}^{\text {rad }}(C(X))=\mathfrak{Z}(C(X))$, as observed in Example 2, but $\mathfrak{Z}(C(X)) \neq \mathfrak{L}(C(X))$ because, in this case, $\mathfrak{L}(C(X))$ is the set of all ideals of $C(X)$. In fact, for the rings $C(X)$, the equality $\mathfrak{L}(C(X))=$ $\mathfrak{Z}(C(X))$ implies $C(X)$ is von Neumann regular. The reason is that $C(X)$ is von Neumann regular if and only if every prime ideal in $C(X)$ is a $z$-ideal ([7, Problem 14B.4.]), and every prime ideal in $C(X)$ is an $\ell$-ideal ([7, Theorem 5.5]).

We observed above that $\mathfrak{Z}^{\infty}(A) \subseteq \mathfrak{Z}^{\text {rad }}(A)$. Three of the examples of $z$-terminating rings we gave above witness that this containment can be an equality. We shall see that the equality $\mathfrak{Z}^{\infty}(A)=\mathfrak{Z}^{\text {rad }}(A)$ is not sufficient for $z$-termination. In so doing, we shall actually exhibit a large class of rings for which the stated equality holds.

Definition 3. A ring $A$ is radically $z$-covered in case $\mathfrak{Z}^{\infty}(A)=\mathfrak{Z}^{\mathrm{rad}}(A)$.

Thus, $A$ is radically $z$-covered precisely when every $\sqrt{z}$-ideal in $A$ is a higher order $z$-ideal. In the following result we show that the class of radically $z$-covered rings includes all Noetherian rings. We doubt that the reader needs to be reminded that a Noetherian ring is a ring in which every ideal is finitely generated. 
Theorem 1. Noetherian rings are radically $z$-covered.

Proof. Let $A$ be a Noetherian ring. We need to show that $\mathfrak{Z}^{\text {rad }}(A) \subseteq \mathfrak{Z}^{\infty}(A)$. So let $I \in \mathfrak{Z}^{\mathrm{rad}}(A)$. Then $\sqrt{I}$ is a $z$-ideal. Since $A$ is Noetherian, there exist finitely many elements $a_{1}, \ldots, a_{n}$ in $A$ such that $\sqrt{I}$ is generated by the set $\left\{a_{1}, \ldots, a_{n}\right\}$. Choose positive integers $k_{1}, \ldots, k_{n}$ such that $a_{i}^{k_{i}} \in I$ for each $i=1, \ldots, n$. Put $k=$ $k_{1}+\cdots+k_{n}$. We claim that $I \in \mathfrak{Z}^{k}(A)$. To show this, consider any $x, y \in A$ with $\mathfrak{M}(x)=\mathfrak{M}(y)$ and $x^{k} \in I$. Then $x \in \sqrt{I}$, and since $\sqrt{I}$ is a $z$-ideal, we deduce that $y \in \sqrt{I}$. Since $\sqrt{I}$ is generated by the elements $a_{1}, \ldots, a_{n}$, there exist elements $u_{1}, \ldots u_{n}$ in $A$ such that $y=u_{1} a_{1}+\cdots+u_{n} a_{n}$. Note that $\left(u_{i} a_{i}\right)^{k_{i}} \in I$, for each $i=1, \ldots, n$. We induct on $n$ to show that $y^{k} \in I$. The result is trivial for $n=1$. So assume $n>1$. For brevity, we write $b_{i}=u_{i} a_{i}$, for $i=1,2, \ldots, n$. Then

$$
\begin{aligned}
\left(b_{1}+\cdots+b_{n}\right)^{k}=\left(b_{1}\right. & \left.+\left(b_{2}+\cdots+b_{n}\right)\right)^{k} \\
= & \sum_{r=0}^{k}\left(\begin{array}{l}
k \\
r
\end{array}\right) b_{1}^{r}\left(b_{2}+\cdots+b_{n}\right)^{k-r} \\
= & \sum_{r<k_{1}}\left(\begin{array}{l}
k \\
r
\end{array}\right) b_{1}^{r}\left(b_{2}+\cdots+b_{n}\right)^{k-r} \\
& +\sum_{r \geq k_{1}}\left(\begin{array}{l}
k \\
r
\end{array}\right) b_{1}^{r}\left(b_{2}+\cdots+b_{n}\right)^{k-r} .
\end{aligned}
$$

The second summand is in $I$ since $b^{k_{1}} \in I$. By the induction hypothesis, each term of the form $\left(b_{2}+\cdots+b_{n}\right)^{k-r}$ is in $I$ if $r<k_{1}$ because then $k-r \geq k_{2}+\cdots+k_{n}$. It follows therefore that $\left(b_{1}+\cdots+b_{n}\right)^{k_{1}+\cdots+k_{n}} \in I$. Thus, $y^{k} \in I$ since $k \geq n$. Therefore $I \in \mathfrak{Z}^{k}(A)$. Consequently, $\mathfrak{Z}^{\text {rad }}(A) \subseteq \mathfrak{Z}^{\infty}(A)$, and hence equality.

Remark 3. Since $\mathbb{Z}$ is Noetherian, it is radically $z$-covered, but it is not $z$-terminating, as observed in Example 5. On the other hand, if $X$ is an infinite set, then $C(X)$ is a radically $z$-covered ring which is not Noetherian.

Remark 4. In the proof of Theorem 1 the fact that $A$ is a Noetherian ring was used to conclude that $\sqrt{I}$ is finitely generated. It may therefore seem as if all we need in the hypothesis is a "weaker condition" than being Noetherian, namely that radical ideals of $A$ be finitely generated. This condition however is equivalent to the ring being Noetherian because every prime ideal is a radical ideal, and a ring in which every prime ideal is finitely generated is actually Noetherian (see, for instance, [11, Theorem 8]). 


\section{DIRECT PRODUCTS AND $z$-TERMINATION}

We now examine the preservation and reflection of the $z$-terminating property by direct products, and by homomorphic images. In the proofs of the following results we shall not decorate the notation $\mathfrak{M}(x)$ with subscripts to indicate where the collection is considered because the element $x$ will make that clear.

Theorem 2. The direct product of finitely many rings is z-terminating if and only if each factor is z-terminating.

Proof. It clearly suffices to prove the assertion for only two rings. To start, let $A$ and $B$ be rings, $I$ and $J$ be ideals of $A$ and $B$, respectively, and $n$ be a positive integer. We claim that

$$
I \times J \text { is a } z^{n} \text {-ideal } \Longleftrightarrow I \text { and } J \text { are } z^{n} \text {-ideals. }
$$

For the left-to-right implication, suppose $a_{1}$ and $a_{2}$ are elements of $A$ with $\mathfrak{M}\left(a_{1}\right)=$ $\mathfrak{M}\left(a_{2}\right)$ and $a_{2}^{n} \in I$. Observe that, in the ring $A \times B, \mathfrak{M}\left(a_{1}, 0\right) \supseteq \mathfrak{M}\left(a_{2}, 0\right)$, because if $\mathfrak{n} \in \mathfrak{M}\left(a_{2}, 0\right)$, then either $\mathfrak{n}=M \times B$ for some $M \in \operatorname{Max}(A)$, or $\mathfrak{n}=A \times N$ for some $N \in \operatorname{Max}(B)$. In the latter case, we clearly have $\left(a_{1}, 0\right) \in \mathfrak{n}$, and in the former we have $a_{2} \in M$, which implies $a_{1} \in M$ since $\mathfrak{M}\left(a_{1}\right)=\mathfrak{M}\left(a_{2}\right)$, hence $\left(a_{1}, 0\right) \in \mathfrak{n}$, so that $\mathfrak{n} \in \mathfrak{M}\left(a_{1}, 0\right)$. Now, $\left(a_{2}, 0\right)^{n} \in I \times J$, and since $I \times J$ is a $z^{n}$-ideal, we have $\left(a_{1}, 0\right)^{n}=\left(a_{1}^{n}, 0\right) \in I \times J$, which implies $a_{1}^{n} \in I$, showing that $I$ is a $z^{n}$-ideal. The proof for $J$ is similar.

For the converse, let $(a, b)$ and $(c, d)$ be elements of $A \times B$ such that $\mathfrak{M}(a, b)=$ $\mathfrak{M}(c, d)$ and $(c, d)^{n} \in I \times J$. Let us show that $\mathfrak{M}(a) \supseteq \mathfrak{M}(c)$. Consider any $M \in$ $\mathfrak{M}(c)$. Then $M \times B$ is a maximal ideal of $A \times B$ containing $(c, d)$, and hence $(a, b)$. Therefore $M \in \mathfrak{M}(a)$, as desired. Since $I$ is a $z^{n}$-ideal, and $c^{n} \in I$, it follows that $a^{n} \in I$. Similarly, $b^{n} \in J$, and hence $(a, b)^{n} \in I \times J$, which proves that $I \times J$ is a $z^{n}$-ideal.

Now suppose $A$ and $B$ are $z$-terminating. Pick positive integers $m$ and $n$ such that

$$
\mathfrak{Z}^{m}(A)=\mathfrak{Z}^{m+1}(A)=\cdots \quad \text { and } \quad \mathfrak{Z}^{n}(B)=\mathfrak{Z}^{n+1}(B)=\cdots .
$$

Say, $m \leq n$. We claim that

$$
\mathfrak{Z}^{n}(A \times B)=\mathfrak{Z}^{n+1}(A \times B)=\cdots .
$$

Let $I \times J \in \mathfrak{Z}^{n+1}(A \times B)$. Then, as proved above, $I \in \mathfrak{Z}^{n+1}(A)$ and $J \in \mathfrak{Z}^{n+1}(B)$, which implies $I \in \mathfrak{Z}^{n}(A)$ and $J \in \mathfrak{Z}^{n}(B)$, whence, again by what we showed above, $I \times J \in \mathfrak{Z}^{n}(A \times B)$. A simple induction argument shows that $\mathfrak{Z}^{n}(A \times B)=\mathfrak{Z}^{n+i}(A \times$ $B$ ) for all $i=1,2, \ldots$. Therefore $A \times B$ is $z$-terminating.

Conversely, suppose $A \times B$ is $z$-terminating. Pick a positive integer $n$ such that

$$
\mathfrak{Z}^{n}(A \times B)=\mathfrak{Z}^{n+i}(A \times B) \text { for all } i=1,2, \ldots
$$


For any $i=1,2, \ldots$, let $I \in \mathfrak{Z}^{n+i}(A)$. Then, the ideal $I \times B$ of $A \times B$ clearly belongs to $\mathfrak{Z}^{n+i}(A \times B)$, and hence to $\mathfrak{Z}^{n}(A \times B)$. Thus, as shown above, $I \in \mathfrak{Z}^{n}(A)$. Therefore $A$ is $z$-terminating. Similarly, $B$ is $z$-terminating.

We have established in the foregoing proof that if $A$ and $B$ are rings, and $n$ is a positive integer, then

$$
\mathfrak{Z}^{n}(A \times B)=\mathfrak{Z}^{n}(A) \times \mathfrak{Z}^{n}(B) .
$$

A moment's reflection, taking into account Lemma 1 and the fact that, for any ideal $I$ of $A$ and any ideal $J$ of $B, \sqrt{I \times J}=\sqrt{I} \times \sqrt{J}$, shows that

$$
\mathfrak{Z}^{\infty}(A \times B)=\mathfrak{Z}^{\infty}(A) \times \mathfrak{Z}^{\infty}(B) \quad \text { and } \quad \mathfrak{Z}^{\mathrm{rad}}(A \times B)=\mathfrak{Z}^{\mathrm{rad}}(A) \times \mathfrak{Z}^{\mathrm{rad}}(B) .
$$

These relations yield the following result.

Proposition 2. The direct product of finitely many rings is radically z-covered if and only if each factor is radically $z$-covered.

Proof. Assume that $A$ and $B$ are radically $z$-covered. Then

$$
\begin{aligned}
\mathfrak{Z}^{\infty}(A \times B) & =\mathfrak{Z}^{\infty}(A) \times \mathfrak{Z}^{\infty}(B) \\
& =\mathfrak{Z}^{\text {rad }}(A) \times \mathfrak{Z}^{\text {rad }}(B)=\mathfrak{Z}^{\text {rad }}(A \times B) .
\end{aligned}
$$

Therefore $A \times B$ is radically $z$-covered. Conversely, assume that $A \times B$ is radically $z$-covered. Then

$$
\begin{aligned}
\mathfrak{Z}^{\infty}(A) \times \mathfrak{Z}^{\infty}(B) & =\mathfrak{Z}^{\infty}(A \times B) \\
& =\mathfrak{Z}^{\mathrm{rad}}(A \times B)=\mathfrak{Z}^{\mathrm{rad}}(A) \times \mathfrak{Z}^{\mathrm{rad}}(B) .
\end{aligned}
$$

Since none of these sets is empty, it follows that $\mathfrak{Z}^{\infty}(A)=\mathfrak{Z}^{\mathrm{rad}}(A)$ and $\mathfrak{Z}^{\infty}(B)=$ $\mathfrak{Z}^{\text {rad }}(B)$; which says $A$ and $B$ are radically $z$-covered.

\section{HOMOMORPHIC IMAGES}

We state upfront that we do not know if $z$-termination is inherited by homomorphic images. We do however have instances of ring homomorphisms that map $z$-terminating (respectively, radically $z$-covered) rings onto rings with the same features. We give such homomorphisms the following name.

Definition 4. A ring homomorphism $\phi: A \rightarrow B$ is strict if it is surjective and it maps non-units to non-units.

Strict homomorphisms abound. We shall present two examples that we shall refer to a little later.

Example 6. Let $R \llbracket x \rrbracket$ denote the ring of formal power series over a ring $R$. The map $\sum_{i=0}^{\infty} a_{i} x^{i} \mapsto a_{0}$ is a strict homomorphism because a power series is a unit in $R \llbracket x \rrbracket$ if and only if $a_{0}$ is a unit in $R$. 
Example 7. Let $A$ be a ring and $I$ be an ideal of $A$. The canonical map $\eta: A \rightarrow A / I$ is strict if and only if $I \subseteq \operatorname{Jac}(A)$. To see this, first assume $I \subseteq \operatorname{Jac}(A)$. For any $a \in A$, if $a+I$ is a unit, then there exists an element $b \in A$ such that $a b+I=1+I$, which implies $1-a b \in I$. Then $1-a b \in \operatorname{Jac}(A)$, so that $a b$ is a unit in $A$, and hence $a$ is a unit in $A$. So $\eta$ maps non-units to non-units. Being surjective, it is therefore strict. Conversely, let $c \in I$, and consider any $x \in A$. Then $\eta(1-x c)=1+I$, which is a unit in $A / I$. Therefore, by hypothesis, $1-x c$ is a unit in $A$, which implies $c \in \operatorname{Jac}(A)$. Therefore $I \subseteq \operatorname{Jac}(A)$.

Although we shall not need the various characterizations of strict homomorphisms, we include them for the sake of completeness. Recall that if $h: R \rightarrow S$ is a surjective ring homomorphism, and $I$ is an ideal in $R$, then $h[I]$ is an ideal in $S$; which may be improper even if $I$ is proper.

Lemma 2. The following are equivalent for a surjective ring homomorphism $\phi: A \rightarrow B$.

(1) $\phi$ is strict.

(2) $\phi[I]$ is a proper ideal in $B$ if I is a proper ideal in $A$.

(3) $\phi[M]$ is a maximal ideal in $B$ if $M$ is a maximal ideal in $A$.

(4) $\phi[M]$ is a proper ideal in $B$ if $M$ is a maximal ideal in $A$.

(5) $\phi^{-1} \phi[M]=M$ if $M$ is a maximal ideal in $A$.

(6) For any $a_{1}, a_{2} \in A, \mathfrak{M}\left(\phi a_{1}\right) \supseteq \mathfrak{M}\left(\phi a_{2}\right)$ implies $\mathfrak{M}\left(a_{1}\right) \supseteq \mathfrak{M}\left(a_{2}\right)$.

Proof. The implications (1) $\Rightarrow(2)$, and (3) $\Rightarrow(4)$ are trivial.

(2) $\Rightarrow$ (3): Let $M$ be a maximal ideal in $A$. By (2), $\phi[M]$ is a proper ideal in $B$, and hence is contained in some maximal ideal $N$, say. Then $M \subseteq \phi^{-1}[N]$, so that $M=\phi^{-1}[N]$, by maximality since $\phi^{-1}[N]$ is a proper ideal. Since $\phi$ is surjective, $N \subseteq \phi \phi^{-1}[N]=\phi[M]$, whence $\phi[M]=N$, a maximal ideal.

(4) $\Rightarrow(5)$ : Let $M$ be a maximal ideal in $A$. Since $M \subseteq \phi^{-1} \phi[M]$, and $\phi^{-1} \phi[M]$ is a proper ideal, by (4), it follows from maximality of $M$ that $M=\phi^{-1} \phi[M]$.

$(5) \Rightarrow(6)$ : Let $a_{1}, a_{2} \in A$ be such that $\mathfrak{M}\left(\phi a_{1}\right) \supseteq \mathfrak{M}\left(\phi a_{2}\right)$. Let $M \in \mathfrak{M}\left(a_{2}\right)$. That is, $M$ is a maximal ideal in $A$ containing $a_{2}$. Then $\phi a_{2} \in \phi[M]$. Since $M=$ $\phi^{-1} \phi[M]$, by (5), it follows that $\phi[M]$ is a proper ideal. Let $N$ be a maximal ideal in $B$ with $\phi[M] \subseteq N$. Then $N \in \mathfrak{M}\left(\phi a_{2}\right)$, and hence, by hypothesis, $N \in \mathfrak{M}\left(\phi a_{1}\right)$. Thus, $a_{1} \in \phi^{-1}[N]$. But now $M=\phi^{-1} \phi[M] \subseteq \phi^{-1}[N]$ implies $M=\phi^{-1}[N]$, so that $M \in \mathfrak{M}\left(a_{1}\right)$, and hence $\mathfrak{M}\left(a_{1}\right) \supseteq \mathfrak{M}\left(a_{2}\right)$.

(6) $\Rightarrow(1)$ : Let $a \in A$ be such that $\phi a$ is a unit. Then $\mathfrak{M}(\phi a)=\varnothing$. So, $\mathfrak{M}(\phi 1) \supseteq$ $\mathfrak{M}(\phi a)$, which, by (6), implies $\mathfrak{M}(1) \supseteq \mathfrak{M}(a)$. But $\mathfrak{M}(1)=\varnothing$; so $a$ is a unit. It follows therefore that $\phi$ maps non-units to non-units.

Next, let us recall from [15, Lemma 1.7] that a ring homomorphism $f: R \rightarrow S$ contracts $z$-ideals to $z$-ideals if and only if it contracts maximal ideals to $z$-ideals. An almost verbatim argument as employed by Mason to prove this establishes the following lemma. 
Lemma 3. Let $\phi: A \rightarrow B$ be a ring homomorphism, and let $n$ be a positive integer. Then the following statements are equivalent.

(1) $\phi^{-1}[I] \in \mathfrak{Z}^{n}(A)$ for every $I \in \mathfrak{Z}^{n}(B)$.

(2) $\phi^{-1}[M] \in \mathfrak{Z}^{n}(A)$ for every $M \in \operatorname{Max}(B)$.

(3) $\phi^{-1}[M] \in \mathfrak{Z}(A)$ for every $M \in \operatorname{Max}(B)$.

(4) $\phi^{-1}[I] \in \mathfrak{Z}(A)$ for every $I \in \mathfrak{Z}(B)$.

Proof. $(1) \Rightarrow(2)$ : This is so because every maximal ideal is a $z$-ideal, and therefore a $z^{n}$-ideal, by the first part of Lemma 1 .

(2) $\Leftrightarrow(3)$ : This follows from the fact that $\phi^{-1}[M]$ is a prime ideal for every maximal ideal $M$, and prime ideals are $z^{n}$-ideals precisely when they are $z$-ideals, by the third part of Lemma 1 .

(3) $\Leftrightarrow(4)$ : This is Mason's cited result.

(2) $\Rightarrow(1)$ : Exactly as in Mason's proof.

As was observed by Mason, surjective ring homomorphisms contract $z$-ideals to $z$-ideals, and therefore satisfy the conditions above which are equivalent to this property.

Now here is the result we are aiming for.

Proposition 3. Let $\phi: A \rightarrow B$ be a strict homomorphism.

(a) If $A$ is z-terminating, then so is $B$.

(b) If $A$ is radically $z$-covered, then so is $B$.

Proof. (a) Let $n$ be a positive integer such that $\mathfrak{Z}^{n}(A)=\mathfrak{Z}^{n+i}(A)$ for every $i=$ $1,2, \ldots$. We aim to show that the same $n$ works for $B$ as well. Fix $i \in \mathbb{N}$, and let $I \in \mathfrak{Z}^{n+i}(B)$. Since $I$ is surjective, $\phi^{-1}[I] \in \mathfrak{Z}^{n+i}(A)$, by Lemma 3 , and hence $\phi^{-1}[I] \in \mathfrak{Z}^{n}(A)$. Consider any $b_{1}, b_{2} \in B$ such that $\mathfrak{M}\left(b_{1}\right) \supseteq \mathfrak{M}\left(b_{2}\right)$ and $b_{2}^{n} \in I$. Pick $a_{1}, a_{2} \in A$ with $\phi a_{1}=b_{1}$ and $\phi a_{2}=b_{2}$. Then $\mathfrak{M}\left(\phi a_{1}\right) \supseteq \mathfrak{M}\left(\phi a_{2}\right)$, which implies $\mathfrak{M}\left(a_{1}\right) \supseteq \mathfrak{M}\left(a_{2}\right)$, by Lemma 2 , since $\phi$ is strict. Now, $a_{2}^{n} \in \phi^{-1}[I]$ implies $a_{1}^{n} \in \phi^{-1}[I]$ since $\phi^{-1}[I]$ is a $z^{n}$-ideal. Consequently, $b_{1}^{n}=\phi\left(a_{1}\right)^{n}=\phi\left(a_{1}^{n}\right) \in I$. This shows that $I$ is a $z^{n}$-ideal, and therefore $\mathfrak{Z}^{n}(B)=\mathfrak{Z}^{n+i}(B)$. Thus, $B$ is $z$ terminating.

(b) Let $I \in \mathfrak{Z}^{\text {rad }}(B)$. Then $\sqrt{I}$ is a $z$-ideal, and therefore $\phi^{-1} \sqrt{I}$ is a $z$-ideal in $A$. But $\phi^{-1} \sqrt{I}=\sqrt{\phi^{-1}[I]}$; so, by hypothesis, there exists a positive integer $n$ such that $\phi^{-1} I \in \mathfrak{Z}^{n}(A)$. Exactly as above, this implies $I \in \mathfrak{Z}^{n}(B)$, which shows that $\mathfrak{Z}^{\mathrm{rad}}(B) \subseteq \mathfrak{Z}^{\infty}(B)$, and hence the desired equality.

Corollary 2. If $A \llbracket x \rrbracket$ is z-terminating (respectively, radically $z$-covered) then $A$ has the same property.

Corollary 3. If $\operatorname{Jac}(A)$ is contained in every higher order $z$-ideal of $A$, then $A$ is $z$-terminating (respectively, radically $z$-covered) if and only if $A / \operatorname{Jac}(A)$ has the same property. 
Proof. For brevity, we write $J=\operatorname{Jac}(A)$. The left-to-right implication always holds since, as observed above, the canonical mapping $A \rightarrow A / J$ is strict.

Conversely, assume that $A / J$ is $z$-terminating. Then there exists a positive integer $n$ such that $\mathfrak{Z}^{n}(A / J)=\mathfrak{Z}^{n+i}(A / J)$ for every $i=1,2, \ldots$. We shall show that

$$
\mathfrak{Z}^{n}(A)=\mathfrak{Z}^{n+1}(A)=\mathfrak{Z}^{n+2}(A)=\cdots .
$$

Fix $i \in \mathbb{N}$, and let $I \in \mathfrak{Z}^{n+i}(A)$. By hypothesis, $J \subseteq I$, and therefore $I / J$ is an ideal of $A / J$. An argument as in the proof of Proposition 3(a) shows that $I / J$ is a $z^{n+i}$-ideal in $A / J$. Hence $I / J \in \mathfrak{Z}^{n}(A / J)$. By Lemma $3, I \in \mathfrak{Z}^{n}(A)$ since $I$ is the inverse image of $I / J$ under the (surjective) canonical map $A \rightarrow A / J$. Therefore $A$ is $z$-terminating.

The proof for the result in parenthesis is similar.

\section{Question}

Is it not the case that, in fact, every homomorphic image of a $z$-terminating ring is $z$-terminating; and similarly for radically $z$-covered rings?

\section{THE $\mathfrak{Z}$-FUNCTORS}

In this section we aim to show that, for each positive integer $n, \mathfrak{Z}^{n}$ can be made into a functor whose domain is a subcategory of Rng ${ }^{\mathrm{op}}$, where Rng denotes the category of (commutative rings) and ring homomorphisms. The subcategory in question is obtained by restricting the morphisms in a suitable manner.

Let $\mathrm{Rng}_{3}$ denote the category whose objects are rings, and whose morphisms are ring homomorphisms $A \stackrel{\phi}{\longrightarrow} B$ that contract $z$-ideals to $z$-ideals. It is routine to check that, indeed, $\mathrm{Rng}_{\mathfrak{z}}$ is a (non-full) subcategory of Rng. For each morphism $A \stackrel{\phi}{\longrightarrow} B$ in $\mathrm{Rng}_{\mathfrak{z}}$, write $B \stackrel{\phi^{*}}{\longrightarrow} A$ for the corresponding morphism in $\mathrm{Rng}_{\mathfrak{z}}{ }^{\mathrm{op}}$.

Theorem 3. For any positive integer $n$ we have the following.

(a) The associations $C \mapsto \mathfrak{Z}^{n}(C)$ and $B \stackrel{\phi^{*}}{\longrightarrow} A \mapsto \mathfrak{Z}^{n}(B) \stackrel{\phi^{-1}}{\longrightarrow} \mathfrak{Z}^{n}(A)$ define $a$ functor $\mathfrak{Z}^{n}:$ Rng $_{\mathfrak{z}}{ }^{\text {op }} \longrightarrow$ Set.

(b) $\mathfrak{Z}^{n}$ preserves finite products.

(c) $\mathfrak{Z}^{n}$ is a subfunctor of $\mathfrak{Z}^{n+1}$.

(d) If $\mathrm{C}$ denotes the subcategory of $\mathrm{Rng}_{3}{ }^{\mathrm{op}}$ whose objects are the rings with zero Jacobson radical, then the functor $\mathrm{T}: \mathrm{C} \rightarrow$ Set, which maps as $\mathfrak{Z}^{n}$, reflects epimorphisms.

Proof. (a) This is verified routinely.

(b) Since our rings have identity, every ideal in $A \times B$ is of the form $I \times J$, for some ideals $I$ and $J$ of $A$ and $B$ respectively. Therefore the assertion in (b) is exactly what was shown in the course of the proof of Theorem 2 that $I \times J$ is a $z^{n}$-ideal if and only if $I$ and $J$ are $z^{n}$-ideals. 
(c) For any object $C$ in $\mathrm{Rng}_{\mathfrak{z}}^{\text {op }}$, we have $\mathfrak{Z}^{n}(C) \subseteq \mathfrak{Z}^{n+1}(C)$, by Lemma 1 . Therefore the mapping $\eta: \operatorname{Obj}\left(\mathrm{Rng}_{\mathfrak{z}}^{\mathrm{op}}\right) \rightarrow \operatorname{Mor}($ Set $)$, given by the inclusion $\eta_{C}: \mathfrak{Z}^{n}(C) \rightarrow$ $\mathfrak{Z}^{n+1}(C)$, is a natural transformation $\eta: \mathfrak{Z}^{n} \rightarrow \mathfrak{Z}^{n+1}$ because, for any morphism $B \stackrel{\phi^{*}}{\longrightarrow} A$ in $\mathrm{Rng}_{\mathfrak{z}}^{\mathrm{op}}$, we have the following commutative diagram:

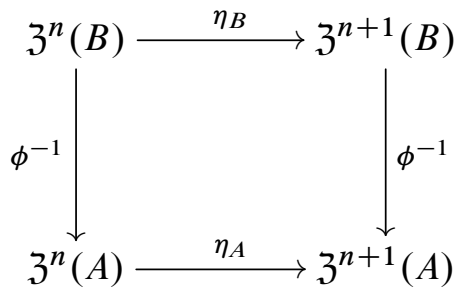

Furthermore, the horizontal morphisms in this diagram are monomorphisms, and so $\mathfrak{Z}^{n}$ is a subfunctor of $\mathfrak{Z}^{n+1}$.

(d) Let $B \stackrel{\phi^{*}}{\longrightarrow} A$ be a morphism in $\mathrm{C}$ for which $\mathrm{T}\left(\phi^{*}\right)$ is an epimorphism. We must show that $\phi^{*}$ is an epimorphism. For this it suffices to show that the ring homomorphism $A \stackrel{\phi}{\longrightarrow} B$ is one-one. Let then $a \in A$ be such that $\phi(a)=0$. Saying the morphism $\mathrm{T}\left(\phi^{*}\right)$ is an epimorphism in Set means that the function $\phi^{-1}: \mathfrak{Z}^{n}(B) \rightarrow$ $\mathfrak{Z}^{n}(A)$ is surjective. Let $M$ be a maximal ideal in $A$. Then $M \in \mathfrak{Z}^{n}(A)$, and hence, by surjectivity of $\phi^{-1}$, there is an ideal $I \in \mathfrak{Z}^{n}(B)$ such that $M=\phi^{-1}[I]$. But now $0=\phi(a) \in I$, which implies $a \in \phi^{-1}[I]=M$. Since $M$ is an arbitrary maximal ideal in $A$, it follows that $a \in \bigcap \operatorname{Max}(A)=\{0\}$ since $A$ has zero Jacobson radical. Therefore $\phi$ is one-one, as required.

The functor T:C $\rightarrow$ Set does not reflect monomorphisms. To give an example substantiating this assertion, we need to know that the inclusion $\iota: \mathbb{Q} \rightarrow \mathbb{R}$ of the field of rational numbers into the field of real numbers is not an epimorphism in Rng. This was brought to our attention by Zurab Janelidze, and we thank him for that. The outline of the validating argument he provided goes as follows.

Let $\mathbb{C}$ be the field of complex numbers. By Galois Theory, $\mathbb{C}$ has only two automorphisms which fix the reals - the identity and the complex conjugate mappings. It is known, however, that $\mathbb{C}$ has infinitely many automorphisms (this is not trivial). Thus, we may consider two maps from $\mathbb{R} \rightarrow \mathbb{C}$ : one, the usual inclusion map, and the other being the composite of the inclusion map with an automorphism of $\mathbb{C}$ which is different from the complex conjugate mapping and is not the identity map. Composing these morphisms with $\iota: \mathbb{Q} \rightarrow \mathbb{R}$ we get equal morphisms since any automorphism of $\mathbb{C}$ fixes the rationals. However, the two maps from $\mathbb{R}$ to $\mathbb{C}$ are not equal (if they were equal, the chosen automorphism would have fixed all reals).

Example 8. The functor T: $\mathrm{C} \rightarrow$ Set does not reflect monomorphisms. The morph$\operatorname{ism} \mathbb{R} \stackrel{\iota^{*}}{\longrightarrow} \mathbb{Q}$ is not a monomorphism in the category $\mathrm{Rng}_{\mathfrak{z}}^{\mathrm{op}}$. However, its image 
$\mathrm{T}\left(\iota^{*}\right): \mathfrak{Z}^{n}(\mathbb{R}) \rightarrow \mathfrak{Z}^{n}(\mathbb{Q})$ is a monomorphism (and, in fact, an isomorphism) in Set because $\mathfrak{Z}^{n}(\mathbb{R})=\{\{0\}, \mathbb{R}\}, \mathfrak{Z}^{n}(\mathbb{Q})=\{\{0\}, \mathbb{Q}\}$, and $\iota^{-1}$ sends $\{0\}$ to $\{0\}$, and $\mathbb{R}$ to $\mathbb{Q}$.

\section{CONCLUDING REMARK}

We had hoped to construct, for each positive integer $n \geq 2$, a $z$-terminating ring $A$ such that the termination commences exactly at stage $n$; that is, such that

$$
\mathfrak{Z}^{n-1}(A) \subset \mathfrak{Z}^{n}(A)=\mathfrak{Z}^{n+1}(A)=\cdots .
$$

This has so far eluded us. We have not pursued the question of sums of higher order $z$-ideals. We leave that for another occasion.

\section{ACKNOWLEDGEMENT}

Thanks are due to the referee for some helpful comments. This paper grew out of an attempt to answer a question that was asked by our colleague, Lungisile Godloza, regarding what we have called $z^{2}$-ideals.

\section{REFERENCES}

[1] D. D. Anderson and J. Kintzinger, "Ideals in direct products of commutative rings," Bull. Austr. Math. Soc., vol. 77, pp. 477-483, 2008, doi: 10.1017/S0004972708000415.

[2] F. Azarpanah and R. Mohamadia, " $\sqrt{z}$-ideals and $\sqrt{z^{\circ}}$-ideals in $C(X)$," Acta Math. Sinica, English Series, vol. 23, no. 6, pp. 989-996, 2006, doi: 10.1007/s10114-005-0738-7.

[3] B. Banaschewski, "On the function rings of pointfree topology," Kyungpook Math. J., vol. 48, pp. 195-206, 2008, doi: 10.5666/KMJ.2008.48.2.195.

[4] B. Banaschewski, "Countable composition closedness and integer-valued continuous functions in pointfree topology," Categor. Gen. Algebraic Struct. Appl., vol. 1, pp. 1-10, 2013.

[5] B. de Pagter, "On $z$-ideals and $d$-ideals in Riesz spaces. III," Indag. Math., vol. 84, no. 4, pp. 409-422, 1981, doi: 10.1016/1385-7258(81)90062-7.

[6] T. Dube and O. Ighedo, "More ring-theoretic characterizations of P-frames," J. Algebra Appl., vol. 14, p. 8, 2015, doi: 10.1142/S0219498815500619.

[7] L. Gillman and M. Jerison, Rings of Continuous Functions, ser. The University Series in Higher Mathematics. New York: Van Nostrand, 1960. doi: 10.1007/978-1-4615-7819-2.

[8] C. B. Huijsman and B. de Pagter, "On $z$-ideals and $d$-ideals in Riesz spaces. I," Indag. Math., vol. 83, no. 2, pp. 183-195, 1980, doi: 10.1016/1385-7258(80)90021-9.

[9] C. B. Huijsman and B. de Pagter, "On z-ideals and $d$-ideals in Riesz spaces. II," Indag. Math., vol. 83, no. 4, pp. 391-408, 1980, doi: 10.1016/1385-7258(80)90040-2.

[10] C. B. Huijsman and B. de Pagter, "Ideal theory in $f$-algebras," Trans. Amer. Math. Soc., vol. 269, pp. 225-245, 1982, doi: 10.2307/1998601.

[11] I. Kaplansky, Commutative Rings. Boston: Allyn and Bacon, 1970.

[12] C. W. Kohls, "Ideals in rings of continuous functions," Fund. Math., vol. 45, pp. 28-50, 1957.

[13] S. Larson, "Convexity conditions on f-rings," Can. J. Math., vol. 38, pp. 48-64, 1986, doi: 10.4153/CJM-1986-003-6.

[14] S. Larson, "Sums of semiprime, $z$, and $d l$-deals in a class of $f$-rings," Proc. Amer. Math. Soc., vol. 109, pp. 895-901, 1990, doi: 10.2307/2048115.

[15] G. Mason, "z-ideals and prime ideals," J. Algebra, vol. 26, pp. 280-297, 1973, doi: 10.1016/00218693(73)90024-0. 
[16] N. McCoy, "Generalized regular rings," Bull. Amer. Math. Soc., vol. 45, pp. 175-178, 1939, doi: 10.1090/S0002-9904-1939-06933-4.

[17] M. A. Mulero, "Algebraic properties of rings of continuous functions," Fund. Math., vol. 144, pp. 55-68, 1996.

[18] S. A. Steinberg, Lattice-ordered rings and modules. New York, London: Springer, 2010. doi: 10.1007/978-1-4419-1721-8.

\section{Authors' addresses}

\section{Themba Dube}

University of South Africa, Department of Mathematical Sciences, P. O. Box 392, 0003 Pretoria, South Africa

E-mail address: dubeta@unisa.ac.za

\section{Oghenetega Ighedo}

University of South Africa, Department of Mathematical Sciences, P. O. Box 392, 0003 Pretoria, South Africa

E-mail address: ighedo@unisa.ac.za 